INPLASY

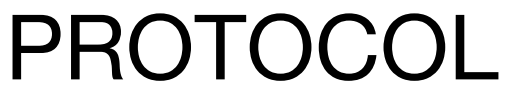

To cite: Lv et al. The prevalence of malnutrition and its effects on the all-cause mortality among patients with heart failure: A systematic review and meta-analysis. Inplasy protocol 202160063. doi:

10.37766/inplasy2021.6.0063

Received: 19 June 2021

Published: 19 June 2021

Corresponding author: Lv Shubin

1286762961@qq.com

Author Affiliation:

The First Affiliated Hospital, and College of Clinical Medicine of Henan University of Science and Technology.

Support: None declared.

Review Stage at time of this submission: Data analysis.

\section{The prevalence of malnutrition and its effects on the all-cause mortality among patients with heart failure: $A$ systematic review and meta-analysis}

Lv, S1; Ru, SC².

Review question / Objective: We conducted a review and meta-analysis of the existing literature to assess the overall prevalence of malnutrition in patients with heart failure and to compare the impact of malnutrition on prognosis in patients with heart failure.

Condition being studied: Heart failure is a common disease, affecting $1 \%-2 \%$ of the global population.Malnutrition is a common complication in patients with chronic heart failure. Epidemiological evidence of malnutrition in patients with heart failure shows that malnutrition often occurs simultaneously with chronic heart failure. The prevalence of malnutrition is higher in patients with heart failure. Malnutrition increases the risk of all-cause mortality in patients with heart failure. Therefore, we should pay attention to the risk of malnutrition in patients with chronic heart failure and reduce the incidence of adverse clinical outcomes.

INPLASY registration number: This protocol was registered with the International Platform of Registered Systematic Review and Meta-Analysis Protocols (INPLASY) on 19 June 2021 and was last updated on 19 June 2021 (registration number INPLASY202160063).

Conflicts of interest:

None declared.

\section{INTRODUCTION}

Review question / Objective: We conducted a review and meta-analysis of the existing literature to assess the overall prevalence of malnutrition in patients with heart failure and to compare the impact of malnutrition on prognosis in patients with heart failure.
Rationale: Malnutrition is common in patients with chronic heart failure. Malnutrition may be related to right ventricular dysfunction and venous congestion, which easily leads to intestinal edema, resulting in malnutrition. We conducted a meta-analysis to assess the prevalence of malnutrition and its impact 
on all-cause mortality in patients with chronic heart failure, and to provide reference for the intervention and prevention of malnutrition in this population.

Condition being studied: Heart failure is a common disease, affecting $1 \%-2 \%$ of the global population. Malnutrition is a common complication in patients with chronic heart failure. Epidemiological evidence of malnutrition in patients with heart failure shows that malnutrition often occurs simultaneously with chronic heart failure. The prevalence of malnutrition is higher in patients with heart failure. Malnutrition increases the risk of all-cause mortality in patients with heart failure. Therefore, we should pay attention to the risk of malnutrition in patients with chronic heart failure and reduce the incidence of adverse clinical outcomes.

\section{METHODS}

Search strategy: Two researchers conducted an extensive and systematic computerized literature review, using the PubMed, Cochrane Library, EMBASE, Medline, CBM, CNKI, WanFang, VIP, and Web of Science databases, using various combinations of the following free text and key terms: "heart failure", "cardiac failure", "HF", "Malnutrition".

Participant or population: Patients With Chronic Heart Failure (12537).

Intervention: Malnutrition.

Comparator: Non malnourished population.

Study designs to be included: Observational studies.

Eligibility criteria: To be included in our review, eligible studies had to (1) be observational studies with cross-sectional or cohort designs analyzing the prevalence of malnutrition and its effects on the allcause mortality among patients with heart failure; (2) diagnose malnutrition according to the clear criteria, which can be divided into malnutrition (risk) and non malnutrition.

Information sources: We searched the available literature in PubMed, Cochrane Library, EMBASE, Medline, CBM, CNKI, WanFang, VIP, and Web of Science databases.

Main outcome(s): (1)The heterogeneity test showed that there was significant heterogeneity among the studies (12 = $83.9 \%, P<0.001)$. Therefore, the random effect model is used.The pooled prevalence of malnutrition among patients with heart failure was $46 \%(95 \%$ Cl $0.43-0.49 ; \mathrm{P}<$ 0.001). (2)Compared with non malnutrition patients, malnutrition patients had a higher all-cause mortality $[\mathrm{HR}=2.15,95 \% \mathrm{Cl}$ $(1.89,2.45)]$, which was as much as two times higher.It means that malnutrition can significantly increase the risk of death, so early intervention can reduce the risk of death in patients with heart failure.

Data management: We use Excel to extract the original data and Endnote to manage the data.

Quality assessment / Risk of bias analysis: Cohort study was given a NewcastleOttawa Scale quality score and Agency for Healthcare Research and Quality was used in the cross-sectional study. Publication bias was assessed using Egger's and/or Begg's tests.

Strategy of data synthesis: Heterogeneity among the included studies was evaluated using the $Q$ test and quantified using 12. An 12 value below $25 \%$ was considered as homogeneity; $25 \%$ to $<50 \%$, low heterogeneity; $50 \%$ to $<75 \%$, moderate heterogeneity ; and at least $75 \%$, substantial heterogeneity. We planned to use a fixed-effects model to meta-analyze pooled data classified as homogeneous or of low heterogeneity, and a random-effects model for data classified as showing moderate or substantial heterogeneity. Publication bias was assessed using Egger's and/or Begg's tests. 
Subgroup analysis: There is almost no heterogeneity among the evaluation criteria of malnutrition. The prevalence rate among the subgroups did not fluctuate much, and remained about $40 \%-50 \%$ overall. There was no significant difference in all-cause mortality among subgroups. The all-cause mortality of malnutrition was about twice as high as that of non malnutrition.

Sensitivity analysis: We performed a sensitivity analysis by omitting the individual studies one at a time to test the robustness of our findings.

Language: Articles in English and chinese were included.

Country(ies) involved: China.

Other relevant information: None.

Keywords: heart failure, cardiac failure, $\mathbf{H F}$, Malnutrition, prevalence, meta-analysis.

Contributions of each author:

Author 1 - Lv Shubin.

Author 2 - Ru SC. 